\title{
DIE ERSTELLUNG VON ONLINE ÜBUNGEN ZUR VERBESSERUNG DES WORTSCHATZES DER DEUTSCHEN SPRACHE
}

\author{
Khadijah Rismayanti Harahap \\ Ahmad Bengar Harahap \\ Rina Evianty
}

\begin{abstract}
AUSZUG
Das Ziel dieser Untersuchung ist, Online Übungen zur Verbesserung des Wortschatzes der deutschen Sprache zu erstellen. In dieser Untersuchung wird die deskriptive qualitative Methode angewendet. Der Prozess der Erstellung von Online Übungen zur Verbesserung des Wortschatzes der deutschen Sprache besteht aus der Erklärung der Phasen von Dick und Carry.Darunter sind : (1) Die Analysephase, (2) Die Designphase, (3) Development oder Entwicklungsphase, (4) Implementation oder Umsetzungsphase, und (5) Evaluation. Die Daten in dieser Untersuchung sind Fragentests aus Studio d A2.Diese Untersuchung wird in einer Klasse der Deutschabteilung an der staatlichen Universität von Medan durchgeführt. Das Ergebnis der Erstellung von Online Übungen zur Verbesserung des Wortschatzes der deutschen Sprache sind Übungen mit der Hot-Potatoes Applikation, die gibt aus JQuiz, JCloze, und JMatch bestehen. Jedes Program enhält Fragen und gibt Feedback, das die Studenten motivieren kann. Die Übungen mit der Hot-Potatoes Applikation können die Studenten Vokabeln lehren. Hoffentlich könnte diese Abschlussarbeit nützlich sein, um unser Wissen zur Wissenschaft über Lernsoftware, vor allem für Deutschlernende anzureichen.

Schlüsselwörter: Online Übungen, Wortschatz, Hot-Potatoes.

\section{EINLEITUNG}

Im Allgemeinen hat die Sprache die Rolle als Bote von einer Person zur anderen. Wenn wir eine Fremdsprache beherrschen, können wir leichter mit anderen kommunizieren. Die deutsch Sprache ist eine der Fremdsprachen, die in der Schule oder an der Universität gelernt wird. Aber nicht alle Leute haben einem Wortschatz der deutschen Sprache. Die meisten Studenten haben Laptops und Smartphones. Sie können mit diesen Medien Online Übungen herunterladen.Dann können sie es online tun, wo immer sie sind.

Während des Studiums benutzen alle Studenten Smartphones für die Kommunikation. Auf Smartphones gibt es viele Anwendungen, zum Beispiel : Facebook, Twitter, Übersetzungsprogramme, und Übungen zum Deutschlernen. Wir können Online-Übungen zum Deutschlernen herunterladen,damit wir die Übungen mit dem Smartphone machen können. Wir können viele Übungen erledigen.
\end{abstract}


DurchApplikationen kann der Wortschatz der Studenten erhöht werden. Es gibt viele Faktoren, diedas Vokabular der Studenten beeinflussen,wie zum Beispiel: Die Studenten interessieren sich weniger dafür die deutsche Sprache zu lernen,öffnen weniger das Wörterbuch, haben keine Internetverbindung oder viele andere Faktoren.

Online-Übungen sind die Anwendungen,die die Studenten für das Training der Fähigkeit verwenden können. Wir können die Applikation“ Hot-Potatoes Programm" verwenden.Hot-Potatoes ist eine Software, um eine Frage Bank zu schaffen. Hot-Potatoes besteht aus 6 Programmen,diese sind : Jquiz, Jcloze, Jmatch, Jmix, Jcross, und The Masher. In dieser Untersuchung werden nur 3 Programme HotPotatoes verwendet. Diese sind Jquiz, Jcloze, und Jmatch. Mit Hot-Potatoes werden für die Untersuchung Online Übungen entwickelt, um die Wortschätze der deutschen Sprache zu verbessern.Die Übungen verwenden das Arbeitsbuch dA2. Die Fragen aus dem Arbeitsbuch bestehen aus Leitpunkten. In der Applikation Hot-Potatoes gibt es keine zeitliche Grenze, um Fragen zu beantworten, und man kann weiter üben, bis man richtig antworten.Nachdem man die Übungen erledigt hat, kann man die Ergebnisse bekommen. Dann bekommt man Noten von dem Online-Übungen. Mit dem geringen Wortschatz der Deutschstudenten wird die Erstellung von Online Übungen mit dem Aplikation "Hot-Potatoes" entwickelt, um die Wortschätze der deutschen Sprache zu verbessern.

\section{THEORETISCHE GRUNDLAGE}

\section{Der Begriff Übungen}

Sukadiyanto (2005: 1) erklärt, dass der Übung im Prinzip ein Prozess ist, der Veränderung zum Besseren ist, die die physikalische Qualität des 7 Funktionsfähigkeit und Qualität Ausrüstung psychischen Körper des Kindes Trainers zu verbessern. Übungen zielen eher auf das wiederholende Training einzelner grammatischer oder lexikalischer Phänomene und deren Automatisierung (Vgl. Klippel, Friederike : 2010).

\section{Der Begriff Aufgaben}


Djamarah und Zain, (1996: 96-97) erklären, dass die Methode derAufgabeeineMethode ist, um das Material zu präsentieren, in dem der Lehrer eine bestimmte Aufgabe stellt, so dass die Schülerzu lernen.

Im Indonesisch Grosser Wörterbuch erklärt weiter, die Aufgabe ist eine Arbeit, die in der Verantwortung einer Person ist.Die Aufgabe istauf den Aufbau neuer Kompetenzen ausgerichtet (Vgl. Klippel, Friederike : 2010).

\section{Begriff Online-Übungen}

Simamorain Kamil (2012:4) stellt fest die Ausbildung als eine Reihe von Aktivitäten, die Fähigkeiten $\mathrm{zu}$ verbessern entworfen, Wissen, Erfahrung oder eine Änderung in der Haltung des Individuums.Hasibuan(2008:69) ausführlich, die Übung ist ein Versuch, das Wissen und Fachkenntnisse eines Mitarbeiters zu tun, um eine bestimmte Aufgabe zu verbessern.

OnlineÜbungen sind deutscheSprachlernübungenmit einemInternetAnschluss.Harsono in Susilo(2012:7) erklärt weiter, dass Training einsystematischer Prozess ist, derimmer wiederwachsendedie wachsende Zahl derTrainingsbelastungerfolgt ist.

Aus den oben genannten Äusserungen kann zusammengefasst werden, dassOnline ÜbungendeutscheSprachlernübungen sind, die eine bestimmte Website oder die Applikation verwenden, diemit dem Internet verbunden ist.

\section{Die Vorteile von Online-Übungen}

Online Übungen sind deutscheSprachlernübungen, die Vorteile und Nachteile haben. Vorteile von Online-Übungen sind :
a. Die Studenten könnenInformationen überdie Wissenschaft bekommen.
b. Die Studenten könnenneueVokabeln lernen.
c. Die Studenten könnendie Website fürdeutscheSprachlern wissen.
d. Die Studenten könnenFragen der Praxisonline ausfüllen, wo immer sie sind.
e. Die Studenten können Vokabular durch die Applikation“ Hot-Potatoes “ üben.
f. Du bist nicht an Zeit und Ort gebunden. Du kannst immer und über all lernen, wie es dir am besten passt.
g. Du musst dich keiner Lerngruppe anpassen. Du bestimmst dein Lerntempo selbst.


h. Lernmaterial zum effizienten Sprachenlernen gibt es für jedes Sprachniveau.

\section{Die Nachteile der Online-Übungen}

Nachteile von Online-Übungen sind :

a. Alle Studenten müssen einSmartphone odereinen Laptop,dem Internet verbindenkönnen.

b. Die Studentenmüssen genug Geld für Online-Übungen haben

c. Der Zugriff auf folgende Studie über das Web ist oft ein Problem für die Lernenden.

d. Die Lernenden können sich schnell müde und gelangweilt fühlen, wenn sie nicht auf die Informationen zugreifen können, weil es keine angemessene Ausrüstung und eine ausreichende Bandbreite gibt.

\section{Der Begriff Wortschatz}

Nugriyantoro (2001 : 146) erklärt, dass Wortschatz der Reichtum an Worten ist, die in einer Sprache gehört. Linse (2005 : 121) erklärt weiter, dass Wortschatz eine Sammlung von Wörtern ist, die jeder kennt. Nach Kridalaksana(2001: 89) heißt es, dass das Vokabular der Wortreichtum von einem Leser oder Schreiber Sprache ist.

Aus den oben genannten Äusserungen kann zusammengefasst werden, dass der Wortschatzein Aspektder eigenen Sprache ist, die einem bestimmten Begriff bezieht. Wortschatz werden für die Informationen verwendet, umInformationenzu empfangen.In dieser Diskussion das Vokabular betreffende Forscherin im deutschen Sprachwortschatz.

\section{Die Bemühungen umden deutschen Wortschatz zu verbessern}

Tarigan, (1994:14) erklärt, Lehrer haben einige Möglichkeiten, umden Wortschatzder Schüler mit Lernaktivitäten zu bereichern. Einige dieser Methoden sind :

1. Deutschstudierende können Synonyme von Wörtern, anonym zu paraphrasieren Worte, Worte auf der Grundlage der gleichen empfehlen.

2. Dozenten könnenden Studenten helfen,die Bedeutung von Wörternund die Satzstrukturzu verstehen.

3. Deutschstudierende können trainieren, um den Wortschatz in einer Vielzahl von Sprachaktivitäten beherrschen. 
Nach Laut Chen (2011:77),gibt es mehr Möglichkeiten Vokabular zu entwickeln, sowohl in der Muttersprache als auch wenn wir eine Fremdsprache lernen, und zwar:

1. Wörter Suchen und eine Liste von Wörtern erstellen, dienotwendig oder wichtig sind.

2. Wir können einen Wörterbuch verwenden, um die schwierigen Vokabeln zu finden

3. Nehmen Sie neue Vokabeln, die Sie nicht verstehen.

4. Schwierige Vokabeln oder neueVokabeln in einem Satz oder einer Phrase notieren.

5. Schwierige Vokabeln sind einfach, wenn man sie richtig nutzen kann.

\section{Der Begriff Hot-Potatos}

Hot-Potatoes ist eine Applikation, um eine Frage Bank zu schaffen.Die Funktion von Hot-Potatoes ist, Material fürwebbasiertes interaktive Lehre zu erstellen.Diese Software wurde von Research Development Teamdi University of Victoria Humanities Computing und Media Center für den Einsatz in der Bildung entwickelt. (Quelle : Pujiastuti,dkk, 2013:117).

Dieses Programm kann unter dieser Adresse https://hotpot.uvic.ca kostenlos bezogen werden.Es gibt sechs Funktionen, die in diesem Programm enthalten sind.Diese sind JQuiz, JCloze, JMatch, JCross, Jmix und Masher. Basierend auf einer Art von Programm Hot Potatoes waren die Forscher die drei Teile des Kurses dieses Programms erklären wollte, das heisst J-Quiz, J-Cloze und J-Match.

\section{Die Arten von Programmen in Hot-Potatoes}

\section{a. JQuiz}

JQuiz ist ein Programm, umAusbildungsmaterial zu entwickeln, das aus vierTypebesteht,diese sind Multiple Choice, Kurzantwort , Hybrid und MultiSelect.

\section{b. JCloze}

J-Cloze ist eine Anwendung für das Schreiben und Abschnitte Bereitstellen montiert werden. Jeder Abschnitt sollte die richtige Antwort enthalten könnte die Möglichkeit, mehr als ein zur Verfügung gestellt werden, einschließlich Richtungen geben, die richtige Antwort zu bekommen.

\section{c. JMatch}


J-Match ist eine Applikation, ein Programm zu machen, die links und rechts entsprechen.Oder es könnte eine Art von Übung oder Fragen zu beantworten sein. Nicht nur das Schreiben, sondern kann auch ein Bild in sie einfügen.

\section{Das Entwicklungsmodell}

Das Entwicklungsmodell wurde von Dick und Carry im Jahr 1996 entwicklt. Es besteht aus Analyse, Design / Konzeption, Development / Entwicklung, Implementation / Umsetzung und Evaluation. Die Funktion des ADDIE-Modells ist eine effektive und effiziente Ausbildung zu entwickeln und zu entwerfen.

\section{Konzeptuelle Grundlage}

In dieser Studie wird eine Anwendung produziert Online-Übungen enthält, um das Vokabular der Deutschstudierenden zu erhöhen. Online Übungen sind Sprachlernübungen, die eine bestimmte Website oder die Applikation verwendet, diemit dem Internet verbunden ist.Die Untersuchung verwendet nur drei Hot-Potatoes Programme. Diese sind JQuiz,JCloze und JMatch. Mit Hot-Potatoes können die Deutschstudierenden ihren Wortschatz erhöhen.

In Hot-Potatoeswird mit drei Programmengelernt, nämlich: JQuiz,JCloze und JMatch. In diesem Fall verwendeten die Forscher die ADDIE Theorie. In der ADDIE Theorie gibt es mehrere Schritteder Entwicklung von Online-Übungen. Von den Online-Übungen wird erwartet, dass sie die Fähigkeiten die Studentenerhöhen.

\section{DAS UNTERSUCHUNGERGEBNIS}

Basierend auf den oben genannten Forschungsmethoden können Forschungsergebnisse erhalten werden. In diesem Kapitel werden die Ergebnisse der Erstellung von Online-Übungen zur Verbesserung des Wortschatzes der deutschen Sprache beschrieben. Jeder Schritt der Untersuchung und die entsprechen Ergebnisse werden in diesem Kapitel erklärt.

\section{Der Prozess der Erstellung von Online Übungen zur Verbesserung des \\ Wortschatzes der deutschen Sprache}

In dieser Untersuchung wird das Untersuchungsmodell von Dick und Carrybenutz. Dieses Modell besteht aus fünf Phasen, nämlich : (1) Analysephase, (2) Design oder Konzeptionsphase (3) Development oder Entwicklungsphase (4) Implementation oder Umsetzung und (5) Evaluationsphase. In diesem Kapitel wird das Ergebnis jeder Phase aufgezeigt. 


\section{Analysephase}

Dies ist die erste Stufe um Online Übungen mit Hot-Potatoes zur Verbesserung des Wortschatzes der deutschen Sprache zu erstellen. In der Analysephase wurden die Informationen über das Lernen der Studenten, die Bedürfnisse und die Lernmedien im Deutschunterricht gesammelt.

\section{Design/Konzeption phase}

In der zweite Stufe werden die Online Übungen mit dem passenden Lernthema entwickelt. Die Untersuchung verwendet die Applikation "Hot-Potatoes" für die Gestaltung. Die Schritte, um Online Übungen zu machen, sind : herunterladen der Applikation "Hot-Potatoes" auf den Computer oder Laptop. Die Übungen werden mit den Programmen JQuiz, JCloze und JMatch mit der Quelle Arbeitsbuch Studio dA2 erstellt.

\section{Development/Entwicklungphase}

In dieser Phase werden alle Mitteln und Materialien vorbereitet, die für die Erstellung Online Übungen verwendet werden sollen, nämlich: der Computer oder Laptop, die Software "Hot-Potatoes", das Arbeitsbuch, Umfrage, Proyektor, CD und Flashdisk.

\section{Implementation/Umsetzungphase}

Die vierte Stufe ist die Umsetzung.Die Umsetzung ist ein konkreter Schritt zur Umsetzung des Lernsystems. Das J-Quiz Programm gibtFeedback, um Studenten zur Beantwortung zu motivieren. Nachdem das Produkt fertig ist, kann es an den Studenten mit Computer oder Laptop getestet werden.In dieser Phase ist die Übung, die gemacht wurde, zu einem Training geworden, das bereit ist, von den Studenten in den drei Programmen der Hot-Potatoesgetestet zuwerden. Das Aussehen des fertigen Trainings ist eine Webseitenanzeige.

\section{Evaluation}

Die Evaluation ist derProzesszusehen, ob das Lernsystem, das entwickelt wurde. Dies ist der letzteSchritt, um gute Online Übungenmitder Hot-Potatoes Software zuerstellen. In dieser Phase werden die Prüfung und die Validierungdurchgeführt. DieseLernmedienwerden von Expertingeprüft. Die 
Testphase würdemit den Deutschstudierenden durchgeführt. Das Programm wurde im Fragebogen gut bewertet. Nach der Meinung von Studenten ist JCloze am schwersten von den drei Programmen des Hot-Potatoes ( JQuiz, JCloze und JMatch ), denn sie muss ihre Antwort im leere Kasten ausfüllen. Die Expertin hat folgende Vorschläge für das Lernmedium :Wennmöglich, kannein Cover hinzugefügt warden und beachte die Erklärung jedes Prozess.Online Übungen mit Hot-Potatoes Software kann über diese Website http://hotpotatoes-dijah.com angesehen werden. Basierend auf der obigenMeinungkannfestgehaltenwerden, dass die Übungen mit der Hot-Potatoes Software als Lernmedium interessant gestaltet sind und besonders gut für die Verbesserung des Wortschatzes der deutschenSprache geeignet sind.

\section{Das Ergebnis der Erstellung von Online Übungen zur Verbesserung des Wortschatzes der deutschen Sprache}

Das Ergebnis der Untersuchung sind Online Übungen zur Verbesserung des Wortschatzes der deutschen Sprache mit der Hot-Potatoes Software. Für diese Übungen wurden mit 3 Programme in Hot-Potatoes verwendet, nämlich JQuiz, JCloze und JMatch. Jedes Programm besteht aus 10 Fragen. Bei der Entwicklung dieser Übungen hat dieForscherin die Studenten getestet. Nachdem die Studenten die Übungen beendet haben, gaben die Forscherin Fragebögen an Studenten über die Übungen in der Hot-Potatoes Software. Der Fragebogen besteht aus 20 Fragen, es gibt auch Kommentare und Vorschläge. Aus dem Fragebogen enhält die Forscherin das Ergebnis, dass die Übungen von den Studenten als gut bewertet werden. Das kann aus den Ergebnissen von Fragebögen, die von Studenten der deutschen Sprache aus gefüllt worden sind, gesehen werden.

Aus dem Fragebogen, der den Studenten gegeben wurde, kommt die Forscherin zu dem Schluss, dass es 13 Punkte von gültigen Fragen und 7 Gegenstände von ungültigen Fragen gibt. So ist das Ergebnis des Prozentsatzes den Studentendaten von 20 Studenten angegeben : es gibt 11 Studenten mit sehr guter Interpretation und 9 Studenten gute Interpretation. Die Online Übungen mit Hot-Potatoes werden auch von einer Expertin getestet. Die Lernmedien sind interessant und sehr schoen für die Studenten. Hot Potatoes werden als Übungsmaterial offline erstellt, können aber auch online sein.

\section{Die Diskussion}


Basierend auf den Ergebnissender Online Übungen zur Verbesserung des Wortschatzes der deutschen Sprache wurde zusammengefasst, dass die Daten in dieser Untersuchung die Fragentests oder die Übungen im Arbeitsbuch sind. In dieser Studie wurde das Dick und Carry Forschungsmodell verwendet. Diese Theorie besteht aus fünf Stufe, nämlich: (1) Analysephase, (2) Designphase,

(3) Die Entwicklungsphase, (4) Die Implementationsphase, und (5) Die Evaluationsphase.

In diesem Kapitel wird jede Phase mit ihrem Ergebnis erklärt. (1) Analysephase. Das ist die erste Stufe. Diese Stufe analysiert die Bedürfnisse der Studenten beim Deutschlernen, die entweder Bücher oder Medien verwenden. (2) bei der Designphase werden die Materialien mit Hot-Potatoes entworfen. (3) In der Entwicklungphase müssen alle Materialien und Ausrüstungen vorbereitet werden. (4) In der Implementation werden alle Fragen im Hot-Potatoes-Programm mit JQuiz, JCloze und JMatch durchgeführt. In dieser Phase ist die Übung, die gemacht wurde, $\mathrm{zu}$ einem Training geworden, das bereit ist, von den Studenten in den drei Programmen der Hot-Potatoes getestet zuwerden. Die Übungen stehen offline bereit. Dann antworten die Studenten über die Verwendung ihres Laptops. (5) Bei der Evaluation werden die Ergebnisse des Programms betrachtet, das wir entwickelt haben. In dieser Phase werden die Prüfung und die Validierung durchgeführt. Der Tests erhielt gute Ergebnisse aus dem Fragebogen, der von Studenten ausgefüllt wurde.

Basierend auf den obigen Erklärungen kann zusammengefasst werden, dass die Erstellung von Online Übungen zur Verbesserung des Wortschatzes der deutschen Sprache mit der ADDIE Theorie sehr gut ist. Sodass die Übungen mit Hot Potatoes als Lernmedium zur Verbesserung des Wortschatzes der deutschen Sprache verwendet werden können.

\section{SCHLUSSFOLGERUNG UND VORSCHLÄGE}

Die Schlussfolgerung

1. Der Prozess der Erstellung von Online Übungen zur Verbesserung des Wortschatzes der deutschen Sprache besteht aus den Phasen von Dick und Carry. Diese sind : (1) Analysephase, (2) Designphase, (3) Entwicklungsphase, (4) Implementationsphase, (5) Evaluationsphase. 
2. Das Ergebnis der Erstellung von Online Übungen zur Verbesserung des Wortschatzes der deutschen Sprache ist :

a. Diese Untersuchung hatOnline Übungen zur Verbesserung des Wortschatzes der deutschen Sprache mit Hot-Potatoes Software erstellt.

b. Die Erstellung von Online Übungen zur Verbesserung des Wortschatzes der deutschen Sprache mit Hot-Potatoes Software wurde von Expertin evaluiert und wurde von Studenten getestet. Hot-Potatoes wurde als Übungsmaterial offline erstellt, aber kann auch online gehen. Aus den Kommentaren der Studierenden im Fragebogen, die gegeben wurden, kommt die Forscherin zu dem Schluss, dass die Übungen mit der Hot-Potatoes Software sehr interessant sind.Hot-Potatoes ist ein interessantes Lernmedium, das dazu genutzt werden kann, Wissen zuerlangen, nicht nur das deutsche Vokabular, sondern auch über die Technologie.

Basierend auf den obigen Meinung kann geschlossen werden, dass die Online Übungen zur Verbesserung des Wortschatzes der deutschen Sprache mit der Hot-Potatoes Software gut sind.

\section{LITERATURVERZEICHNIS}

Chen, Febe. 2011. Competence for Success 1 Maksimalkan Potensi Diri Menjadi Professional Berkompeten Keterampilan dan Pengetahuan. PT.Gramedia Pustaka Utama, Jakarta

Funk, Herman. 2013. Studio d A2 Deutsch als Fremdsprache Kurs- und Übungsbuch. Katalis, Jakarta.

Götz, Dieter dkk. 2003. Langenscheidt Großwörterbuch deutsch als Fremdsprache. Berlin dan München: Langenscheidt KG.

Häussermann,Ulrich,Hans-Eberhard Piepho. 1996. Aufgaben-Handbuch Deutsch als Fremdsprache. Iudicium, München.

Klippel, Friederike (2010), S. 316: „Übung“. In: Surkamp, Carola (Hrsg.): Metzler Lexikon Fremdsprachendidaktik. Stuttgart: Metzler. 314-31

Linse, Caroline T. 2005. Practical English Language Teaching: Young Learners. New York: Mc Graw-Hill 
Studia: Journal des Deutschsprogramms p-ISSN 2301-6108 e-ISSN 2654-9573

https://jurnal.unimed.ac.id/2012/index.php/studia/index

Nurgiyantoro,Burhan. 2001. Penilaian dalam Pengajaran Bahasa dan Sastra. Yogyakarta: BPFE.

Pribadi, Benny. 2014. Desain dan Pengembangan Program Pelatihan Berbasis Kompetensi-Implementasi Model ADDIE. Prenada Media Grup, Jakarta

Pujiastuti,Suci, dkk. 2013. Komputer dan Multimedia untuk Pengajaran dan Pembelajaran Bahasa Jerman. Unimed Press, Medan

Rusman dkk. 2011. Pembelajaran Berbasis Teknologi Informasi dan Komunikasi. PT. Raja Grafindo Persada, Jakarta

Soedjito dan Djoko Saryono. (2011). Kosakata Bahasa Indonesia. Aditya Media Publishing

Wicke,Rainer (2014). Aufgaben,Übungen,Interaktion. Deutsch Lehren Lernen 4. München: Klett-Langenscheidt.

https://id.pinterest.com/explore/online-\%C3\%BCbungen-deutsch-934631722299/ gelesen am 10.November 2016, um 15.00 Uhr.

http://www.deutsch-lernen.com/learn-german-online/beginners/lesson_1.php gelesen am 23. Dezember 2016, um 10.00 Uhr.

http://xportal.klett-sprachen.de/aspekte/ gelesen am 5 Januari 2017, um 13.00 Uhr. http://jurnalonline.um.ac.id/data/artikel/.pdf http://www.bildungsdoc.de/infos/weiterbildung/sprachen-online-lernen http://repository.unhas.ac.id/bitstream/handle/.pdf

Khadijah Rismayanti Harahap is an alumni of German language study program of Unimed, Medan 
Studia: Journal des Deutschsprogramms p-ISSN 2301-6108 e-ISSN 2654-9573

https://jurnal.unimed.ac.id/2012/index.php/studia/index 
